\title{
Src homology domain 2 adaptors affect adherence of Salmonella enterica serovar Typhimurium to non- phagocytic cells
}

\author{
Correspondence \\ B. Brett Finlay \\ bfinlay@interchange.ubc.ca
}

Received 29 March 2007

Revised 15 June 2007

Accepted 26 June 2007
Erin C. Boyle, ${ }^{1,2}$ Nat F. Brown, ${ }^{1}$ John H. Brumell ${ }^{4,5}$ and B. Brett Finlay ${ }^{1,2,3}$

\author{
${ }^{1}$ Michael Smith Laboratories, University of British Columbia, Vancouver, BC V6T 1Z4, Canada \\ ${ }^{2}$ Department of Microbiology and Immunology, University of British Columbia, Vancouver, \\ BC V6T 1Z4, Canada \\ ${ }^{3}$ Department of Biochemistry and Molecular Biology, University of British Columbia, Vancouver, \\ BC V6T 1Z4, Canada \\ ${ }^{4}$ Cell Biology Program, Hospital for Sick Children, Toronto, ON M5G 1X8, Canada \\ ${ }^{5}$ Department of Molecular and Medical Genetics, University of Toronto, Toronto, ON M5S 1A8, \\ Canada
}

The ability of Salmonella enterica serovar Typhimurium ( $S$. Typhimurium) to penetrate the intestinal epithelium is key to its pathogenesis. Bacterial invasion can be seen as a two-step process initially requiring adherence to the host cell surface followed by internalization into the host cell.

Evidence suggests that adherence of $S$. Typhimurium to host cells is receptor-mediated; however, the host cell receptor(s) has/have not been identified. Internalization of $S$. Typhimurium absolutely requires the actin cytoskeleton yet only a few of the cytoskeletal components involved in this process have been identified. In order to identify host proteins that may play a role in $S$. Typhimurium invasion, the recruitment of actin-associated proteins was investigated. The contribution of recruited Src homology 2 adaptor proteins to invasion was further investigated and it was found that, while not involved in bacterial internalization itself, the adaptors Nck and ShcA influenced adherence of $S$. Typhimurium to non-phagocytic cells.

\section{INTRODUCTION}

Salmonella enterica serovar Typhimurium (S. Typhimurium) is a facultative intracellular pathogen that gains entry into the host through oral ingestion of contaminated food or water. It is a major cause of human gastroenteritis and, in mice, serves as a model for typhoid fever. Critical for $S$. Typhimurium virulence is its ability to attach to mucosal surfaces in the intestine and induce its own uptake into epithelial cells. Upon adherence to the host cell surface, $S$. Typhimurium usurps the host cell cytoskeleton, initiating the formation of localized actin-driven lamellipodia-like extensions that facilitate internalization of bacteria into membrane-bound vacuoles (Schlumberger \& Hardt, 2006).

Abbreviations: CFTR, cystic fibrosis transmembrane conductance regulator; FAK, focal adhesion kinase; LPP, lipoma preferred partner; MEF, mouse embryonic fibroblast; RTK, receptor tyrosine kinase; $\mathrm{SH}, \mathrm{Src}$ homology; siRNA, small interfering RNA; SPI-1, Salmonella pathogenicity island 1 .

A supplementary figure showing recruitment of actin-associated proteins to sites of $S$. Typhimurium invasion, a supplementary table listing host proteins investigated for their recruitment to sites of $\mathrm{S}$. Typhimurium invasion, and details of the methods used to produce this data are available with the online version of this paper.
In the context of disease, bacterial adherence and internalization are means by which pathogens colonize the host, spread to systemic sites, and evade both host defence mechanisms and antibiotic treatment. Accordingly, understanding the molecular mechanisms involved in these events is of the utmost importance.

Adherence to the host cell surface is considered to be a prerequisite for internalization of $S$. Typhimurium into non-phagocytic cells. In general, adherence involves the engagement of host cell receptors by bacterial adhesins. $S$. Typhimurium possess a surprisingly large number of fimbrial (Baumler et al., 1997) and non-fimbrial adhesins (Dorsey et al., 2005; Gerlach et al., 2007; Kingsley et al., 2002; Latasa et al., 2005). The cell surface receptor(s) for $S$. Typhimurium has/have been characterized as trypsin- and neuraminidase-sensitive (Finlay et al., 1989). Therefore, adherence of $S$. Typhimurium is probably a receptormediated phenomenon, but surprisingly little is known about the host determinants that participate in this interaction. The closely related pathogen, Salmonella enterica serovar Typhi, but not $S$. Typhimurium, uses the cystic fibrosis transmembrane conductance regulator (CFTR) as a receptor for attachment to epithelial cells 
(Pier et al., 1998). The host cell determinants required for $S$. Typhimurium adherence remain unknown.

Subsequent to adherence, $S$. Typhimurium is internalized into non-phagocytic cells, a process that involves a complex interaction between bacterial and host factors. Salmonella pathogenicity island 1 (SPI-1) encodes genes required for internalization as well as a type 3 secretion system (T3SS) (Galan \& Curtiss, 1989; Mills et al., 1995). T3SSs are needle-like protein complexes that mediate the translocation of specific proteins (effectors) from the bacteria directly into the host cell's cytoplasm (Ghosh, 2004). The SPI-1 type 3-secreted effectors SopE and SopE2 directly stimulate Rho GTPases by acting as exchange factors (Friebel et al., 2001; Hardt et al., 1998; Stender et al., 2000; Zhou et al., 2001). Another SPI-1 type 3-secreted effector, SopB (also known as SigD), stimulates SH3containing guanine nucleotide exchange factor, an exchange factor for RhoG (Patel \& Galan, 2006). Rac1 and RhoG are key regulators of the actin cytoskeleton and their activation by SopB, SopE and SopE2 is required for internalization of $S$. Typhimurium into epithelial cells (Criss et al., 2001; Friebel et al., 2001; Hardt et al., 1998; Patel \& Galan, 2006; Stender et al., 2000; Zhou et al., 2001). Stimulation of Rho GTPases by $S$. Typhimurium leads to the recruitment and activation of N-WASP (Unsworth et al., 2004) and WAVE (Shi et al., 2005). N-WASP and WAVE are known activators of the Arp2/3 complex, which is essential for $S$. Typhimurium internalization into polarized (Criss \& Casanova, 2003) and non-polarized epithelial cells (Criss \& Casanova, 2003; Unsworth et al., 2004). Accordingly, N-WASP and WAVE have also been shown to participate in $S$. Typhimurium internalization (Shi et al., 2005; Unsworth et al., 2004). More recently, the focal adhesion proteins p130cas and focal adhesion kinase (FAK) have also been shown to be essential for invasion of S. Typhimurium (Shi \& Casanova, 2006).

Src homology 2 (SH2) domains bind specifically to phosphorylated tyrosine residues (Buday, 1999; Pawson, 2007). SH2-containing proteins mediate protein-protein interactions and act as adaptors that modulate the actin cytoskeleton and signal transduction, often linking receptor tyrosine kinases (RTKs) to downstream effectors (Buday, 1999; Pawson, 2007). Interestingly, during infection of host cells, $\mathrm{SH} 2$ adaptors are often exploited by viral and bacterial pathogens. For example, interaction between the $\mathrm{SH} 2$ adaptor Nck and the vaccinia virus protein A36R facilitates actin-based motility of the virus (Frischknecht et al., 1999). Enteropathogenic Escherichia coli inserts its own receptor, translocated intimin receptor (Tir), into the host cell plasma membrane, and the binding of Nck to Tir mediates actin pedestal formation underneath the bacterium (Gruenheid et al., 2001). In addition, the adaptor Crk is involved in invasion of Shigella and Yersinia into epithelial cells (Bougneres et al., 2004; Bruce-Staskal et al., 2002; Burton et al., 2003). Whether S. Typhimurium targets $\mathrm{SH} 2$ adaptors during invasion has not been explored.
The major objective of this study was to identify novel host components involved in invasion of $S$. Typhimurium into non-phagocytic cells. We examined this early hostpathogen interface by assessing the recruitment of various actin-associated proteins to the site of $S$. Typhimurium invasion. The contribution of $\mathrm{SH} 2$ adaptors to bacterial invasion was further investigated and an important early role for the adaptors Nck and ShcA in the adherence of $S$. Typhimurium to host cells was identified.

\section{METHODS}

Bacterial strains and cell lines. Wild-type $S$. Typhimurium SL1344 and its isogenic mutant strain, $\Delta s o p B / E / E 2$, have been described previously (Boyle et al., 2006; Wray \& Sojka, 1978). Bacteria were grown in Luria-Bertani (LB) medium supplemented with $100 \mu \mathrm{g}$ streptomycin $\mathrm{ml}^{-1}$ or $25 \mu \mathrm{g}$ kanamycin $\mathrm{ml}^{-1}$ where appropriate. HeLa cells (CCL-2) were obtained from the American Type Culture Collection Cell Biology Stock Center (Manassas, VA, USA). Mouse embryonic fibroblast (MEF) cell lines generated from $\mathrm{Nck} 1+/$ $+; \mathrm{Nck} 2+/+(\mathrm{Nck} 1+/ \mathrm{Nck} 2+), \quad \mathrm{Nck} 1-/-; \mathrm{Nck} 2+/+$ (Nck1-/ $\mathrm{Nck} 2+), \quad \mathrm{Nck} 1+/+; \mathrm{Nck} 2-/-(\mathrm{Nck} 1+/ \mathrm{Nck} 2-)$ and $\mathrm{Nck} 1-/$ - ;Nck2-/- (Nck1-/Nck2-) embryos were kindly provided by Tony Pawson (Samuel Lunenfeld Research Institute, Toronto, ON, Canada) and have been described previously (Bladt et al., 2003; Gruenheid et al., 2001). Nck1-/Nck2- cells complemented with wild-type human Nck1 or a dominant-negative version of Nck1 with inactivating mutations in all three SH3 domains (Kall) were kindly provided by Tony Pawson (Samuel Lunenfeld Research Institute) and have been described previously (Rivera et al., 2006; Tanaka et al., 1995). Cell lines were grown in Dulbecco's minimal Eagle's medium (DMEM) supplemented with $10 \%(\mathrm{v} / \mathrm{v})$ fetal calf serum (DMEM/ FCS) at $37{ }^{\circ} \mathrm{C}$ in a humidified atmosphere with $5 \% \mathrm{CO}_{2}$.

Infection of cultured cells. Cells were seeded into 24 -well plates with or without $12 \mathrm{~mm}$-diameter coverslips at a density of $1.0 \times 10^{5}$ cells $\mathrm{ml}^{-1} 24 \mathrm{~h}$ prior to infection. Bacteria were grown for $16 \mathrm{~h}$ at $37{ }^{\circ} \mathrm{C}$ with shaking and then subcultured $(1: 33)$ in LB broth for $3 \mathrm{~h}$. The bacterial inoculum was prepared by pelleting bacteria at $10000 \mathrm{~g}$ for $2 \mathrm{~min}$ and then resuspending them in PBS, pH 7.4. The inoculum was diluted in DMEM/FCS and added to cells at an m.o.i. of $\sim 50$ for $10 \mathrm{~min}$ at $37^{\circ} \mathrm{C}$.

Immunofluorescence. Ten minutes after infection, coverslips were
washed three times with PBS and fixed with $2.5 \%(\mathrm{v} / \mathrm{v})$ paraformalde-
hyde for 20 min at room temperature. After fixation, the coverslips
were washed three times with PBS and the cells were permeabilized
with $0.1 \%$ (v/v) Triton X-100 in PBS. Following permeabilization, the
cells were blocked with $10 \%$ (v/v) normal goat serum in PBS and
probed with rabbit polyclonal anti-CrkI/II (BD BioSciences), anti-ShcA
(Upstate Biotechnology), anti-Nck (a kind gift from T. Pawson, Samuel
Lunenfeld Research Institute) and mouse monoclonal anti-Salmonella
LPS (BioDesign). Following incubation with primary antibodies, the
cells were washed three times with $0.1 \%$ Triton X-100 in PBS and
probed with Alexa- 488 and Cy5 dye-conjugated antibodies and Alexa-
568 -conjugated phalloidin to detect actin (all antibodies supplied by
Molecular Probes). The coverslips were mounted on glass slides in
Mowiol (Aldrich) and samples were viewed on a Zeiss Axiovert S100
TV microscope attached to a Bio-Rad Radiance Plus confocal scanhead.
Confocal sections were projected using ImageJ v.1.31 and were
imported into Adobe Photoshop v.7.0.

Plasmids and transfection. The Nck1-GFP construct was kindly provided by Tony Pawson. Plasmid DNA was prepared using Qiagen 
columns and was transfected into cells using Fugene 6 (Roche) in accordance with the manufacturer's instructions. Cells were transfected $18 \mathrm{~h}$ prior to infection.

siRNA. Small interfering RNA (siRNA) SMARTpool (Dharmacon RNA Technologies) sequences targeting CrkI/II or ShcA, or nontargeting scrambled control siRNA were diluted and stored according to the manufacturer's instructions. The CrkI/II- and ShcA-targeting sequences have been published previously (Kisielow et al., 2002; Nagashima et al., 2002). HeLa cells were transfected $24 \mathrm{~h}$ after seeding with 40 pmol siRNA per well using Lipofectamine 2000 (Invitrogen) according to the manufacturer's instructions. Cells were trypsinized and seeded $48 \mathrm{~h}$ post-transfection; bacterial infections were carried out $72 \mathrm{~h}$ post-transfection, since knockdown of ShcA and Crk protein levels was maximal at this time point (see below and Fig. 2b).

Immunoblotting. Cells were washed three times with PBS and lysed in NP buffer $[20 \mathrm{mM}$ Tris/ $\mathrm{HCl}, \mathrm{pH} 7.5,150 \mathrm{mM} \mathrm{NaCl}, 1 \%(\mathrm{v} / \mathrm{v})$ Nonidet P-40, $10 \mathrm{mM} \mathrm{Na}_{4} \mathrm{P}_{2} \mathrm{O}_{7}, 50 \mathrm{mM} \mathrm{NaF}$ ] supplemented with a Complete protease inhibitor tablet (Roche). The protein concentration of each sample was quantified using a bicinchoninic acid assay (Sigma). An equal amount of total protein $(15 \mu \mathrm{g})$ was loaded in each lane of the gel. Samples were electrophoresed through a $12 \%$ SDSpolyacrylamide gel and transferred onto nitrocellulose membrane (Millipore). The membranes were incubated for $1 \mathrm{~h}$ at room temperature in blocking buffer [Tris-buffered saline, $0.1 \%(\mathrm{v} / \mathrm{v})$ Tween 20 (TBST), $5 \%(\mathrm{w} / \mathrm{v})$ skimmed milk] and then incubated overnight at $4{ }^{\circ} \mathrm{C}$ with primary antibodies diluted in blocking buffer. After washing in TBST, the membranes were incubated with the appropriate HRP-conjugated secondary antibodies diluted in blocking buffer for $1 \mathrm{~h}$ at room temperature. After washing in TBST, the bands were detected using an enhanced chemiluminescence (ECL) kit (Amersham) according to the manufacturer's instructions and quantified using ImageJ v.1.36b software.

Gentamicin protection assay. After a $10 \mathrm{~min}$ infection, cells were washed three times with PBS and incubated with DMEM/FCS for $20 \mathrm{~min}$ at $37{ }^{\circ} \mathrm{C}$. Thirty minutes post-infection, the medium was replaced with DMEM/FCS containing $50 \mu$ gentamicin $\mathrm{ml}^{-1}$. Two hours post-infection, cells were washed three times with PBS and lysed in PBS containing $1 \%$ Triton X-100 and $0.1 \%(\mathrm{w} / \mathrm{v})$ SDS. Bacterial dilutions were made in PBS and plated onto LB agar for enumeration of bacterial c.f.u.

Inside/outside immunostaining. Cells were infected for $10 \mathrm{~min}$, washed three times with PBS, fixed with $2.5 \%$ paraformaldehyde, and blocked in $10 \%$ normal goat serum in PBS. Cells were probed with polyclonal anti-Salmonella LPS, washed with PBS, and incubated with Alexa 568-conjugated donkey anti-rabbit antibodies to label the bacteria outside the cells. Cells were washed with PBS and permeabilized with $0.1 \%$ Triton X-100 in PBS. Cells were probed with rabbit polyclonal anti-Salmonella LPS, washed with $0.1 \%$ Triton X-100 in PBS, and incubated with an Alexa-350-conjugated donkey anti-rabbit antibody. Coverslips were mounted as described above and viewed on a Zeiss Axiophot epifluorescence microscope. Twenty fields of view were selected at random and analysed for each experiment. 'Internalization' was defined as the number of intracellular bacteria per cell while 'adherence' was defined as the number of intracellular and cell-associated bacteria per cell. 'Internalization efficiency' was calculated as: (Internalization/Adherence) $\times 100$.

Statistical analysis. Data were analysed using Prism 4.0. One-way ANOVAs and Student's unpaired $t$-tests were performed using a $95 \%$ confidence interval. For ANOVAs, Bonferroni tests were applied post hoc.
RESULTS

\section{Recruitment of actin-associated proteins to sites of $\mathbf{S}$. Typhimurium invasion}

In order to identify new host proteins that may play a role in $S$. Typhimurium invasion, the recruitment of actinbinding proteins, focal adhesion proteins and proteins associated with actin polymerization was investigated. Many of the proteins assessed for recruitment to sites of $S$. Typhimurium invasion were chosen because they have important functional roles in lamellipodia formation and cell motility and therefore may be important for Salmonella-induced ruffles and bacterial invasion. $S$. Typhimurium-induced membrane ruffles are defined as sites of coincidence of F-actin accumulation, membrane extension, and bacterial adherence. Recruitment of these proteins to $S$. Typhimurium-induced membrane ruffles was visualized using immunofluorescent staining of epithelial cells infected for $10 \mathrm{~min}$. Of the proteins screened, the following were recruited to $S$. Typhimuriuminduced membrane ruffles: $\alpha$-actinin, ezrin, LPP, profilin, talin, tropomyosin, VASP, WIP and zyxin (see Supplementary Fig. S1 and Supplementary Table S1, available with the online version of this paper). As shown previously (Finlay et al., 1991), vinculin did not accumulate at sites of invasion (Fig. S1). Recruitment of SH2 adaptors was investigated as they are key regulators of the actin cytoskeleton. The SH2 adaptors Nck, Crk and ShcA were all recruited to $S$. Typhimurium-induced membrane ruffles (Fig. 1). These data demonstrate the extent to which $S$. Typhimurium engages the host cell cytoskeleton and reveal that the SH2 adaptors Nck, Crk and ShcA are recruited during the invasion process.

\section{Nck and ShcA, but not Crk, affect the extent of invasion of $S$. Typhimurium into non-phagocytic cells}

A functional role for $\mathrm{SH} 2$ adaptors during $S$. Typhimurium invasion has, to our knowledge, not been previously explored. The SH2 adaptor Nck is strongly recruited to Salmonella-induced ruffles (Fig. 1) and therefore its role during invasion was investigated. Mammals possess two Nck family members, Nck1/ $\alpha$ and Nck2/ $\beta$ (also called Grb4) (Braverman \& Quilliam, 1999). Gentamicin-protection assays were performed using mouse embryonic fibroblasts deficient in Nck1 and/or Nck2 to determine the requirement for different Nck family members in $S$. Typhimurium invasion. It is of note that $S$. Typhimurium invades to a similar extent into fibroblasts and epithelial cells (data not shown). Compared to Nck1 +/Nck2 + cells, the number of intracellular $S$. Typhimurium was significantly reduced in cells lacking both Nck1 and Nck2 (Fig. $2 \mathrm{a} ; P<0.001)$. However, the absence of either Nck1 or Nck2 did not significantly reduce the number of intracellular S. Typhimurium (Fig. 2a), demonstrating that Nck1 and Nck2 can each functionally compensate for the absence 

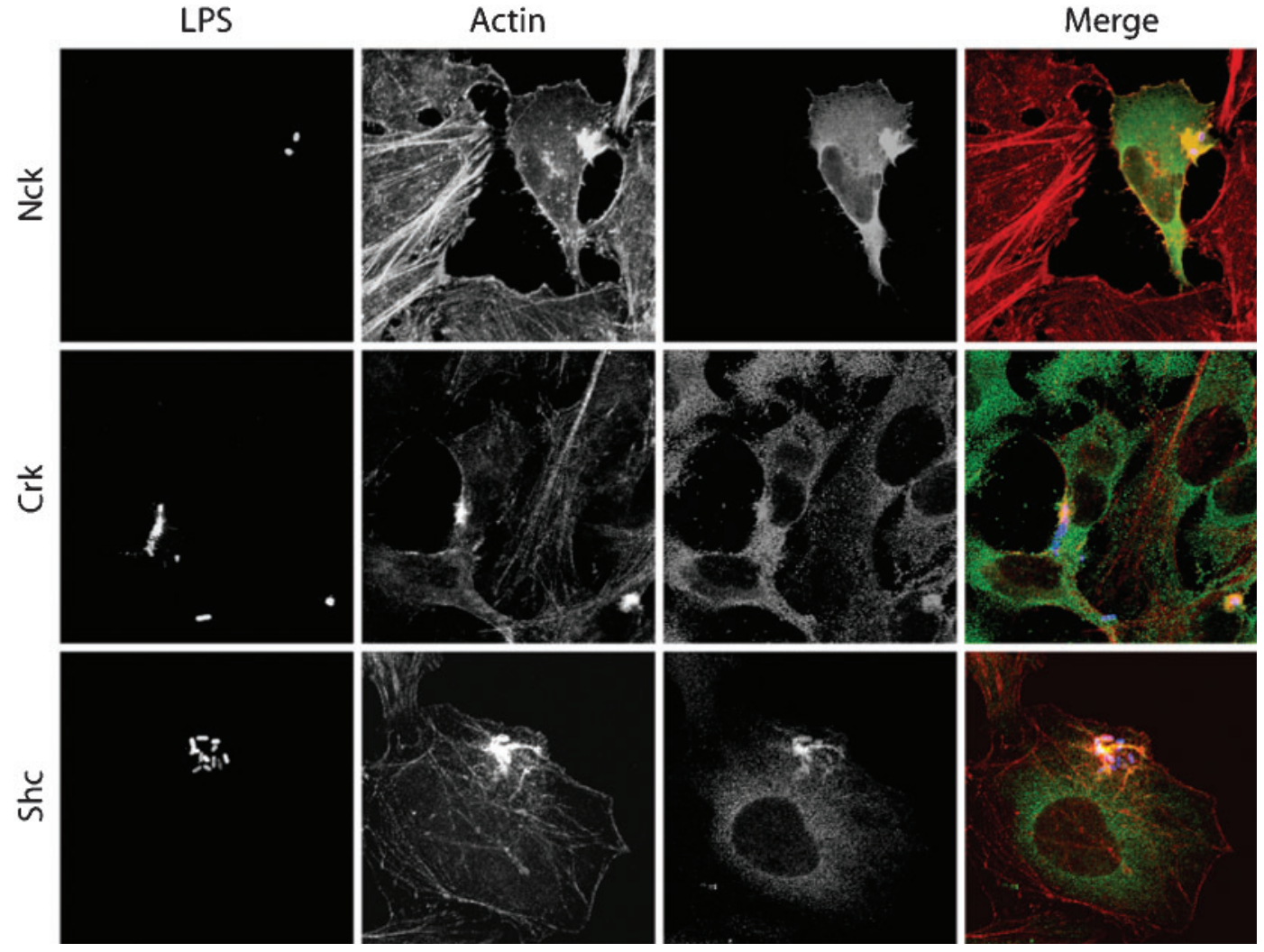

Fig. 1. The SH2 adaptors Nck, Crk and ShcA are recruited to sites of S. Typhimurium invasion. Nck-GFP was transfected into HeLa cells $18 \mathrm{~h}$ prior to infection. HeLa cells were infected with wild-type S. Typhimurium for 10 min and stained with phalloidin to label actin (red), an anti-LPS antibody (blue) to label bacteria, and antibodies towards endogenous Crk or ShcA (green). Images are en face Z-projections of confocal slices and are representative of results from at least three different experiments.

of the other. These data reveal that Nck1 and Nck2 play an important role in the invasion process of $S$. Typhimurium.

A functional role for the recruited SH2 adaptors Crk and ShcA was also explored. Alternate splicing of the human $c r k$ gene generates two Crk proteins; CrkI, consisting of an SH2 domain and an SH3 domain, and CrkII, which has an additional C-terminal SH3 domain (Buday, 1999). ShcA exists in three isoforms, namely p66, p52 and p46, which differ only in their $\mathrm{N}$-terminal regions (Ravichandran, 2001). Using commercially available siRNA directed towards sequences of ShcA and Crk that were common to all isoforms, expression of all isoforms of ShcA and Crk were successfully knocked down (Fig. 2b). Importantly, expression of ShcA and Crk were not affected when cells were transfected with scrambled (control) siRNA (Fig. 2b). We infected siRNA-treated cells with $S$. Typhimurium $72 \mathrm{~h}$ post-transfection, as knockdown of ShcA and Crk was maximal at that time point (Fig. 2b). Invasion of $S$. Typhimurium was quantified using the gentamicin protection assay. While knockdown of Crk did not affect the extent of invasion, knockdown of ShcA significantly increased the level of $S$. Typhimurium invasion $(P<0.001$; Fig. 2c). These results suggest that ShcA inhibits the invasion process of $S$. Typhimurium.

\section{Nck and ShcA affect adherence of S. Typhimurium to non-phagocytic cells}

The extent of invasion can be influenced by changes in bacterial adherence to the cell surface and/or by changes in the ability of bacteria to trigger actin-dependent internalization. In order to distinguish between these two possibilities, cells were infected with wild-type $S$. Typhimurium and intracellular and extracellular (cellassociated) bacteria were quantified. Compared to Nck1 + I Nck2 + cells, $S$. Typhimurium adhered significantly less to Nck1-/Nck2 - cells (Fig. 3a; $P<0.001$ ). In order to confirm that the inability of $S$. Typhimurium to adhere to Nck1-/Nck2 - cells was attributable to specific deletion of the $n c k$ genes, we performed complementation analysis. Adherence of $S$. Typhimurium to Nck1-/Nck2- cells expressing Nck1 was significantly greater than adherence to Nck1-/Nck2 - cells expressing a dominant-negative form of Nck1 (Kall; Fig. 3a; $P<0.001$ ). Therefore, Nck does indeed contribute to the adherence of $S$. Typhimurium to non-phagocytic cells. Conversely, in cells where ShcA expression had been knocked down, bacterial adherence significantly increased (Fig. 3b, $P<0.0001$ ), demonstrating that ShcA inhibits this early interaction of $S$. Typhimurium with host cells. 
(a)

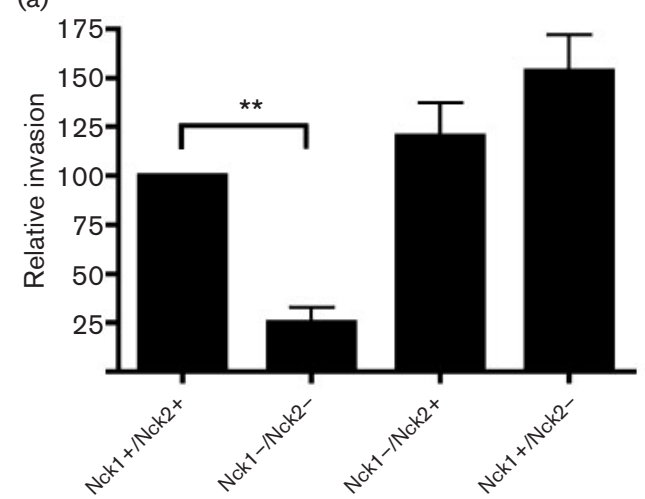

(b)

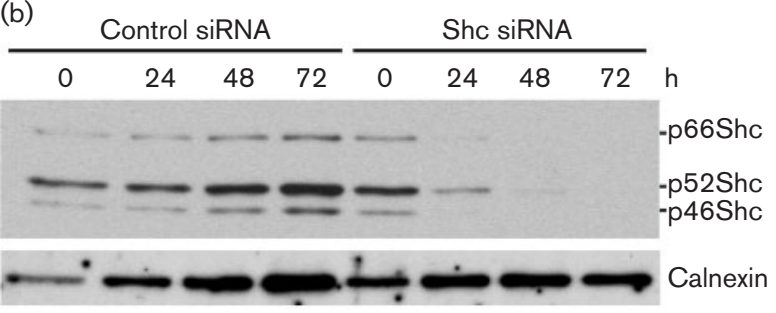

Control siRNA

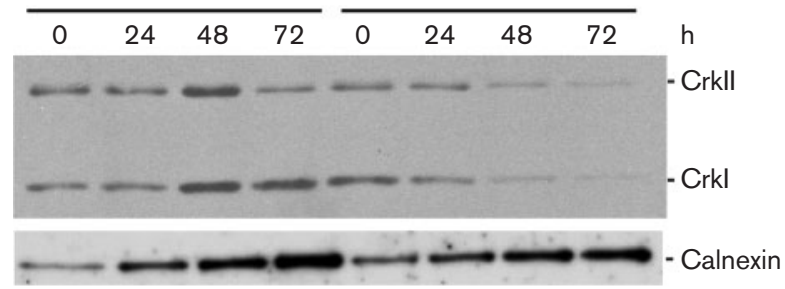

(c)

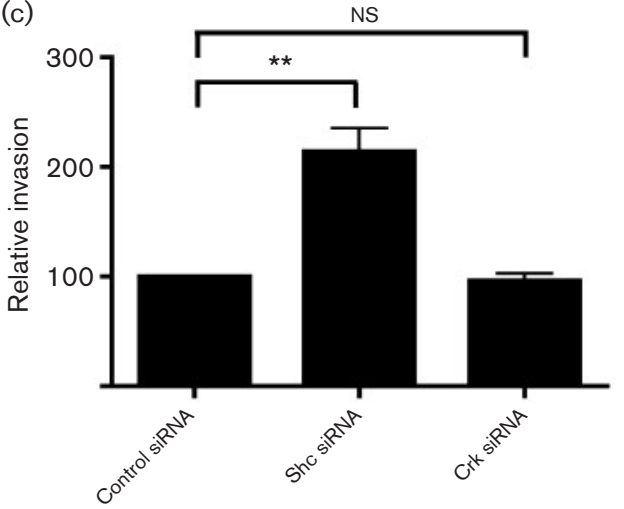

Fig. 2. Nck and ShcA, but not Crk, affect the extent of invasion of $S$. Typhimurium into non-phagocytic cells. (a) Nck1+/Nck2+, Nck1-/Nck2-, Nck1+/Nck2- and Nck1-/Nck2+ MEFs were infected with wild-type $S$. Typhimurium for $10 \mathrm{~min}$ and invasion was quantified using a gentamicin protection assay. Invasion is reported as a percentage normalized to invasion into Nck1+l Nck2 + cells and values are expressed as means \pm SEM of three different experiments, each performed in duplicate. ${ }^{* *}, P<0.001$ using a Bonferroni post-hoc test. (b) HeLa cells were transfected with either scrambled (control), anti-ShcA, or anti-Crkl/ll siRNA and assessed at various time points post-transfection for expression of ShcA or Crkl/ll by Western immunoblotting. A corresponding calnexin immunoblot is shown as a loading control. (c) HeLa cells were transfected with either scrambled (control), anti-ShcA or anti-Crkl/ll siRNA and infected with wild-type $S$. Typhimurium $72 \mathrm{~h}$ post-transfection. Invasion was quantified using a gentamicin-protection assay. Invasion is reported as a percentage normalized to invasion into control siRNA-transfected cells and values are expressed as means \pm SEM of three different experiments, each performed in duplicate. NS, Not significant; ${ }^{*}, P<0.001$ using a Bonferroni post-hoc test.

we also investigated whether Nck was recruited to sites of attachment using the invasion-defective $S$. Typhimurium mutant, $\Delta$ sopB/E/E2. After a 10 min infection, Nck was not recruited to sites where the invasion-defective mutant adhered (Fig. 3e).

\section{Nck and ShcA do not affect internalization efficiency of $S$. Typhimurium into non-phagocytic cells}

Because Nck and ShcA could affect both bacterial adherence and internalization, we investigated whether these adaptors also influenced internalization efficiency, defined here as the proportion of adherent bacteria that are internalized. The internalization efficiency of $S$. Typhimurium into Nck1-/Nck2- $(44.4 \pm 6.0 \%)$ cells did not significantly differ from that for wild-type cells $(42.8 \pm 5.1 \%$; not significant, Student's $t$-test). Similarly, internalization efficiency into ShcA knockdown cells $(46.3 \pm 2.5 \%)$ was not significantly different from control siRNA-treated cells $(56.5 \pm 3.7 \%$; not significant, Student's $t$-test). Therefore, Nck and ShcA do not play significant roles in internalization in that, once bound to the cell surface, $S$. Typhimurium can be internalized to a similar extent with or without these SH2 adaptors. Therefore, the differences in invasion observed in Nck1-/Nck2- and Shc knockdown cells (Fig. 2a, c) are probably solely attributable to differences in the ability of $S$. Typhimurium to adhere to host cells.

\section{DISCUSSION}

The ability of $S$. Typhimurium to penetrate the intestinal epithelium is key to its pathogenesis. Bacterial invasion can be seen as a two-step process first requiring adherence to 

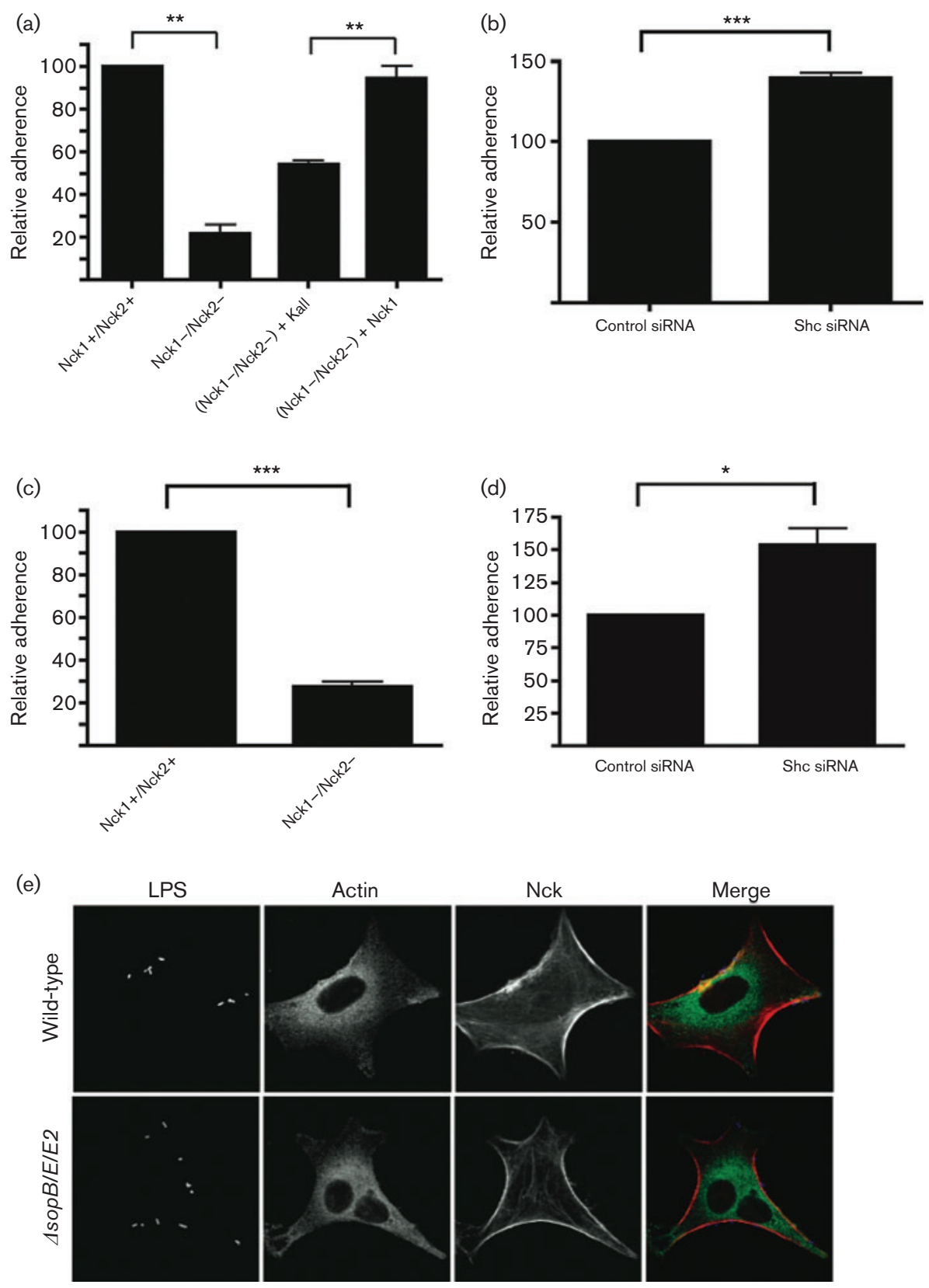

the host cell surface followed by internalization into the host cell. Evidence suggests that adherence of $S$. Typhimurium to host cells is receptor-mediated (Finlay et al., 1989); however, the host cell receptor(s) has/have not been identified. Uptake of $S$. Typhimurium is absolutely dependent on the actin cytoskeleton yet few of the cytoskeletal components involved have been identified. In order to identify new host determinants involved in $S$. Typhimurium invasion, the recruitment of various actinassociated proteins to the site of invasion was investigated. Initial characterization of Salmonella-induced-ruffle components revealed a surprising variety of actin-associated proteins recruited upon infection, including actin-binding proteins, $\mathrm{SH} 2$ adaptors, focal adhesion proteins and proteins involved in actin polymerization. To test whether recruited proteins had functional roles during invasion, this study focused on the contribution of $\mathrm{SH} 2$ adaptor proteins.

SH2- and SH3-domain-containing adaptor proteins facilitate protein-protein interactions (Buday, 1999). SH2 domains bind specific phosphorylated tyrosine residues while SH3 domains bind proline-rich sites. This study examined the involvement of the recruited $\mathrm{SH} 2$ adaptors Nck, Crk and ShcA during S. Typhimurium invasion. The SH2-SH3 adaptor Nck often links RTKs with the actin cytoskeleton and is best known for its roles in growth factor signalling, cell motility and integrin signalling 
Fig. 3. Nck and ShcA affect adherence of $S$. Typhimurium to non-phagocytic cells. (a) MEFs $[$ Nck1 +/Nck2 +, Nck1-/Nck2-, Nck1-/Nck2- expressing Nck1, and Nck1-/Nck2- expressing dominant-negative Nck1 (Kall)] were infected for 10 min with wild-type $S$. Typhimurium and adherence was quantified using inside/outside immunostaining. Adherence is reported as a percentage normalized to adherence to Nck1 +/Nck2 + cells; values are means \pm SEM of three experiments, each performed in duplicate. ${ }^{* *}, P<0.001$ using a Bonferroni post-hoc test. (b) HeLa cells were transfected with either scrambled (control) or antiShcA siRNA and infected with wild-type S. Typhimurium $72 \mathrm{~h}$ post-transfection. Adherence was quantified using inside/outside immunostaining and is reported as a percentage normalized to adherence to control siRNA-transfected cells; values are means \pm SEM of three experiments, each performed in duplicate. ${ }^{* \star *}, P<0.0001$ using a Student's unpaired $t$-test. (c) Nck1 $+/$ $\mathrm{Nck} 2+$ and Nck1-/Nck2- MEFs were infected for $10 \mathrm{~min}$ with the invasion-defective mutant, $\Delta s o p B / E / E 2$. Adherence was quantified by immunostaining and is reported as a percentage normalized to adherence to Nck1+/Nck2 + cells; values are means \pm SEM of three replicates. ${ }^{* \star *}, P<0.0001$ using a Student's unpaired $t$-test. (d) HeLa cells were transfected with either scrambled (control) or anti-ShcA siRNA and infected with the invasion-defective mutant, $\Delta$ sopB/E/E2, $72 \mathrm{~h}$ post-transfection. Adherence was quantified by immunostaining and is reported as a percentage normalized to adherence to control siRNAtransfected cells; values are means \pm SEM of three replicates. ${ }^{*}, P<0.01$ using a Student's unpaired $t$-test. (e) Recruitment of Nck to sites of adherence was assessed by infecting HeLa cells for 10 min with wild-type S. Typhimurium or the invasiondefective mutant, $\triangle s o p B / E / E 2$. Cells were stained with phalloidin to label actin (red), an anti-LPS antibody (blue) to label bacteria, and an antibody toward Nck (green). Images are en face Z-projections of confocal slices and are representative of at least three different experiments.

(Buday et al., 2002). We were initially interested in investigating the role of $\mathrm{Nck}$ as it is a potent upstream regulator of N-WASP and WAVE (Eden et al., 2002; Rohatgi et al., 2001; Tomasevic et al., 2007), both of which are important for S. Typhimurium invasion (Shi et al., 2005; Unsworth et al., 2004). Nck was recruited to sites of $S$. Typhimurium invasion and was required for efficient invasion by affecting bacterial adherence to the host cell surface. In these studies, Nck1 and Nck2 were found to be functionally redundant, which is consistent with other studies (Bladt et al., 2003; Braverman \& Quilliam, 1999).

The SH2-SH3 adaptor Crk is involved in cell migration, signalling pathways from RTKs and invasion of the bacterial pathogens Yersinia and Shigella into epithelial cells (Bougneres et al., 2004; Bruce-Staskal et al., 2002; Buday, 1999; Burton et al., 2003). Although enriched in Salmonella-induced ruffles, Crk was not required in the invasion process. Some of the proteins recruited to sites of $S$. Typhimurium invasion may not perform a functional role at this site but are rather recruited because their binding partners are actively recruited to ruffles. In addition, one might suspect there is a certain amount of functional redundancy among recruited proteins that could compensate in the absence of Crk.

The SH2 adaptor ShcA is best known for its roles in signalling events controlling cell proliferation, survival and apoptosis, and has been implicated in signalling via many different types of receptors including growth factor receptors, antigen receptors, G-protein-coupled receptors, hormone receptors, cytokine receptors and integrins (Ravichandran, 2001). ShcA has been linked to cytoskeletal organization of cells by regulating integrin-mediated random cell migration $(\mathrm{Gu}$ et al., 1999). Interestingly, the recruitment of ShcA to sites of $S$. Typhimurium invasion appeared to inhibit adherence of $S$. Typhimurium to epithelial cells.

Because Nck and ShcA affect S. Typhimurium adherence, they are clearly affecting the host cell surface. One way Nck and ShcA could affect the host cell surface is through modulating protein expression. Nck1 has been shown to enhance protein translation through its interaction with eukaryotic initiation factor 2 (eIF2) (Kebache et al., 2002, 2004; Latreille \& Larose, 2006). Accordingly, perhaps Nck is involved in the expression of the $S$. Typhimurium receptor. Alternatively, Nck and ShcA could affect the composition of the cell surface by influencing the trafficking of the $S$. Typhimurium receptor(s) to and/or from the cell surface. The cell surface pool of any given receptor can be influenced by internalization of the receptor into endocytic vesicles (endocytosis) and the redistribution of internalized receptors back to the plasma membrane (endocytic recycling) (Soldati \& Schliwa, 2006). Is there evidence that $\mathrm{Nck}$ and Shc are involved in receptor trafficking? The actin cytoskeleton actively participates in the recycling of certain G-protein-coupled receptors, the transferrin receptor, glucose transporter 4 and CFTR (Ganeshan et al., 2007; Jiang et al., 2002; Stanasila et al., 2006; Yan et al., 2005). Accordingly, Nck and Shc could affect receptor trafficking through their interactions with the actin cytoskeleton. For example, N-WASP has recently been shown to be critical for surface localization of CFTR by affecting both endocytosis and endocytic recycling of CFTR (Ganeshan et al., 2007). Because Nck is a potent upstream regulator of N-WASP (Tomasevic et al., 2007) it may also be involved in these processes. The actin cytoskeleton is also known to actively participate in the transport and fusion of secretory vesicles to the plasma membrane (Malacombe et al., 2006). Nck localizes to certain endo- and exocytic vesicles (Benesch et al., 2002) and recent evidence suggests that Nck is required for actinbased translocation of exocytic vesicles to the cell surface (Lettau et al., 2006).

The ability of cytoplasmic proteins to directly or indirectly regulate the affinity of receptors for their ligands is referred to as 'inside-out signalling'. Once thought to be exclusive to integrin activation (Ginsberg et al., 2005), inside-out 
signalling has also been characterized for Fc (Bracke et al., 2000) and hormone (Caunt et al., 2004) receptor activation. Could Nck modulate adherence of $S$. Typhimurium through its influence on the activation state of a cell surface receptor? Nck has been implicated in inside-out signalling through its involvement in integrin activation (Becker et al., 2000). However, because Nck is not recruited to sites of adherence of an invasion-defective mutant (Fig. 3e), Nck is more likely to affect the cell surface through receptor trafficking or expression.

The Arp $2 / 3$ complex, N-WASP, WAVE, FAK and p130cas have all been shown to be involved in invasion of $S$. Typhimurium into non-phagocytic cells (Criss \& Casanova, 2003; Shi et al., 2005; Shi \& Casanova, 2006; Unsworth et al., 2004). With the exception of the WAVE study, these studies have not addressed whether the observed decreases in invasion were attributable to decreases in $S$. Typhimurium attachment. We dissected the two factors that could contribute to differences in the number of intracellular bacteria: adherence and internalization. Although the role of Nck and ShcA in bacterial adherence was unexpected, future studies should address the role of additional cytoskeletal proteins in S. Typhimurium adherence. Significantly, this is apparently the first study to identify host molecules involved in $S$. Typhimurium adherence, and although this involvement is presumably indirect, these findings may provide clues as to the identity of the cell surface receptor for $S$. Typhimurium. Clearly more work must to be done in order to identify the mechanism by which Nck and ShcA modulate the host cell surface. In addition, this study has identified many new actin-associated proteins recruited to sites of $S$. Typhimurium invasion that may play important roles during the invasion process. Understanding the molecular mechanisms underlying bacterial adherence and internalization is key to understanding how $S$. Typhimurium causes disease.

\section{ACKNOWLEDGEMENTS}

The authors would like to thank Tony Pawson for providing Nck reagents and Danika Goosney and Guntram Grassl for helpful discussions. Work in B. B. F.'s laboratory is supported by operating grants from the Canadian Institutes of Health Research (CIHR) and the Howard Hughes Medical Institute (HHMI). N. F. B. is a Michael Smith Foundation for Health Research postdoctoral fellow. B. B. F. is a CIHR Distinguished Investigator, an HHMI International Research Scholar and the UBC Peter Wall Distinguished Professor. J. H. B. holds an Investigators in Pathogenesis of Infectious Disease Award from the Burroughs Wellcome Fund, a Premier's Research Excellence award from the Ontario Ministry of Economic Development and Trade and a Boehringer Ingelheim (Canada) Young Investigator Award in Biological Sciences.

\section{REFERENCES}

Baumler, A. J., Tsolis, R. M. \& Heffron, F. (1997). Fimbrial adhesins of Salmonella typhimurium. Role in bacterial interactions with epithelial cells. Adv Exp Med Biol 412, 149-158.
Becker, E., Huynh-Do, U., Holland, S., Pawson, T., Daniel, T. O. \& Skolnik, E. Y. (2000). Nck-interacting Ste20 kinase couples Eph receptors to c-Jun $\mathrm{N}$-terminal kinase and integrin activation. Mol Cell Biol 20, 1537-1545.

Benesch, S., Lommel, S., Steffen, A., Stradal, T. E., Scaplehorn, N., Way, M., Wehland, J. \& Rottner, K. (2002). Phosphatidylinositol 4,5biphosphate (PIP2)-induced vesicle movement depends on N-WASP and involves Nck, WIP, and Grb2. J Biol Chem 277, 37771-37776.

Bladt, F., Aippersbach, E., Gelkop, S., Strasser, G. A., Nash, P., Tafuri, A., Gertler, F. B. \& Pawson, T. (2003). The murine Nck SH2/ SH3 adaptors are important for the development of mesodermderived embryonic structures and for regulating the cellular actin network. Mol Cell Biol 23, 4586-4597.

Bougneres, L., Girardin, S. E., Weed, S. A., Karginov, A. V., OlivoMarin, J. C., Parsons, J. T., Sansonetti, P. J. \& Van Nhieu, G. T. (2004). Cortactin and Crk cooperate to trigger actin polymerization during Shigella invasion of epithelial cells. J Cell Biol 166, 225-235.

Boyle, E. C., Brown, N. F. \& Finlay, B. B. (2006). Salmonella enterica serovar Typhimurium effectors SopB, SopE, SopE2 and SipA disrupt tight junction structure and function. Cell Microbiol 8, 1946-1957.

Bracke, M., Nijhuis, E., Lammers, J. W., Coffer, P. J. \& Koenderman, L. (2000). A critical role for PI 3-kinase in cytokine-induced F $\alpha$-receptor activation. Blood 95, 2037-2043.

Braverman, L. E. \& Quilliam, L. A. (1999). Identification of Grb4/ $\operatorname{Nck} \beta$, a Src homology 2 and 3 domain-containing adapter protein having similar binding and biological properties to Nck. J Biol Chem 274, 5542-5549.

Bruce-Staskal, P. J., Weidow, C. L., Gibson, J. J. \& Bouton, A. H. (2002). Cas, Fak and Pyk2 function in diverse signaling cascades to promote Yersinia uptake. J Cell Sci 115, 2689-2700.

Buday, L. (1999). Membrane-targeting of signalling molecules by SH2/SH3 domain-containing adaptor proteins. Biochim Biophys Acta 1422, 187-204.

Buday, L., Wunderlich, L. \& Tamas, P. (2002). The Nck family of adapter proteins: regulators of actin cytoskeleton. Cell Signal 14, 723-731.

Burton, E. A., Plattner, R. \& Pendergast, A. M. (2003). Abl tyrosine kinases are required for infection by Shigella flexneri. EMBO J 22, 5471-5479.

Caunt, C. J., Hislop, J. N., Kelly, E., Matharu, A. L., Green, L. D., Sedgley, K. R., Finch, A. R. \& McArdle, C. A. (2004). Regulation of gonadotropin-releasing hormone receptors by protein kinase C: inside out signalling and evidence for multiple active conformations. Endocrinology 145, 3594-3602.

Criss, A. K. \& Casanova, J. E. (2003). Coordinate regulation of Salmonella enterica serovar Typhimurium invasion of epithelial cells by the Arp2/3 complex and Rho GTPases. Infect Immun 71, 2885-2891.

Criss, A. K., Ahlgren, D. M., Jou, T. S., McCormick, B. A. \& Casanova, J. E. (2001). The GTPase Rac1 selectively regulates Salmonella invasion at the apical plasma membrane of polarized epithelial cells. J Cell Sci 114, 1331-1341.

Dorsey, C. W., Laarakker, M. C., Humphries, A. D., Weening, E. H. \& Baumler, A. J. (2005). Salmonella enterica serotype Typhimurium MisL is an intestinal colonization factor that binds fibronectin. Mol Microbiol 57, 196-211.

Eden, S., Rohatgi, R., Podtelejnikov, A. V., Mann, M. \& Kirschner, M. W. (2002). Mechanism of regulation of WAVE1-induced actin nucleation by Rac1 and Nck. Nature 418, 790-793.

Finlay, B. B., Heffron, F. \& Falkow, S. (1989). Epithelial cell surfaces induce Salmonella proteins required for bacterial adherence and invasion. Science 243, 940-943. 
Finlay, B. B., Ruschkowski, S. \& Dedhar, S. (1991). Cytoskeletal rearrangements accompanying Salmonella entry into epithelial cells. $J$ Cell Sci 99, 283-296.

Friebel, A., Ilchmann, H., Aepfelbacher, M., Ehrbar, K., Machleidt, W. \& Hardt, W. D. (2001). SopE and SopE2 from Salmonella typhimurium activate different sets of RhoGTPases of the host cell. J Biol Chem 276, 34035-34040.

Frischknecht, F., Moreau, V., Rottger, S., Gonfloni, S., Reckmann, I., Superti-Furga, G. \& Way, M. (1999). Actin-based motility of vaccinia virus mimics receptor tyrosine kinase signalling. Nature 401, 926-929.

Galan, J. E. \& Curtiss, R., III (1989). Cloning and molecular characterization of genes whose products allow Salmonella typhimurium to penetrate tissue culture cells. Proc Natl Acad Sci U S A 86, 6383-6387.

Ganeshan, R., Nowotarski, K., Di, A., Nelson, D. J. \& Kirk, K. L. (2007). CFTR surface expression and chloride currents are decreased by inhibitors of N-WASP and actin polymerization. Biochim Biophys Acta 1773, 192-200.

Gerlach, R. G., Jackel, D., Stecher, B., Wagner, C., Lupas, A., Hardt, W. D. \& Hensel, M. (2007). Salmonella Pathogenicity Island 4 encodes a giant non-fimbrial adhesin and the cognate type 1 secretion system. Cell Microbiol 9, 1834-1850.

Ghosh, P. (2004). Process of protein transport by the type III secretion system. Microbiol Mol Biol Rev 68, 771-795.

Ginsberg, M. H., Partridge, A. \& Shattil, S. J. (2005). Integrin regulation. Curr Opin Cell Biol 17, 509-516.

Gruenheid, S., DeVinney, R., Bladt, F., Goosney, D., Gelkop, S., Gish, G. D., Pawson, T. \& Finlay, B. B. (2001). Enteropathogenic E. coli Tir binds Nck to initiate actin pedestal formation in host cells. Nat Cell Biol 3, 856-859.

Gu, J., Tamura, M., Pankov, R., Danen, E. H., Takino, T., Matsumoto, K. \& Yamada, K. M. (1999). Shc and FAK differentially regulate cell motility and directionality modulated by PTEN. J Cell Biol 146, 389-403.

Hardt, W. D., Chen, L. M., Schuebel, K. E., Bustelo, X. R. \& Galan, J. E. (1998). S. typhimurium encodes an activator of Rho GTPases that induces membrane ruffling and nuclear responses in host cells. Cell 93, 815-826.

Jiang, Z. Y., Chawla, A., Bose, A., Way, M. \& Czech, M. P. (2002). A phosphatidylinositol 3-kinase-independent insulin signaling pathway to N-WASP/Arp2/3/F-actin required for GLUT4 glucose transporter recycling. J Biol Chem 277, 509-515.

Kebache, S., Zuo, D., Chevet, E. \& Larose, L. (2002). Modulation of protein translation by Nck-1. Proc Natl Acad Sci U S A 99, 5406-5411.

Kebache, S., Cardin, E., Nguyen, D. T., Chevet, E. \& Larose, L. (2004). Nck-1 antagonizes the endoplasmic reticulum stress-induced inhibition of translation. J Biol Chem 279, 9662-9671.

Kingsley, R. A., Santos, R. L., Keestra, A. M., Adams, L. G. \& Baumler, A. J. (2002). Salmonella enterica serotype Typhimurium ShdA is an outer membrane fibronectin-binding protein that is expressed in the intestine. Mol Microbiol 43, 895-905.

Kisielow, M., Kleiner, S., Nagasawa, M., Faisal, A. \& Nagamine, Y. (2002). Isoform-specific knockdown and expression of adaptor protein ShcA using small interfering RNA. Biochem J 363, 1-5.

Latasa, C., Roux, A., Toledo-Arana, A., Ghigo, J. M., Gamazo, C., Penades, J. R. \& Lasa, I. (2005). BapA, a large secreted protein required for biofilm formation and host colonization of Salmonella enterica serovar Enteritidis. Mol Microbiol 58, 1322-1339.

Latreille, M. \& Larose, L. (2006). Nck in a complex containing the catalytic subunit of protein phosphatase 1 regulates eukaryotic initiation factor $2 \alpha$ signaling and cell survival to endoplasmic reticulum stress. J Biol Chem 281, 26633-26644.

Lettau, M., Qian, J., Linkermann, A., Latreille, M., Larose, L., Kabelitz, D. \& Janssen, O. (2006). The adaptor protein Nck interacts with Fas ligand: guiding the death factor to the cytotoxic immunological synapse. Proc Natl Acad Sci U S A 103, 5911-5916.

Malacombe, M., Bader, M. F. \& Gasman, S. (2006). Exocytosis in neuroendocrine cells: new tasks for actin. Biochim Biophys Acta 1763, $1175-1183$.

Mills, D. M., Bajaj, V. \& Lee, C. A. (1995). A $40 \mathrm{~kb}$ chromosomal fragment encoding Salmonella typhimurium invasion genes is absent from the corresponding region of the Escherichia coli K-12 chromosome. Mol Microbiol 15, 749-759.

Nagashima, K., Endo, A., Ogita, H., Kawana, A., Yamagishi, A., Kitabatake, A., Matsuda, M. \& Mochizuki, N. (2002). Adaptor protein Crk is required for ephrin-B1-induced membrane ruffling and focal complex assembly of human aortic endothelial cells. Mol Biol Cell 13, 4231-4242.

Patel, J. C. \& Galan, J. E. (2006). Differential activation and function of Rho GTPases during Salmonella-host cell interactions. J Cell Biol 175, 453-463.

Pawson, T. (2007). Dynamic control of signaling by modular adaptor proteins. Curr Opin Cell Biol 19, 112-116.

Pier, G. B., Grout, M., Zaidi, T., Meluleni, G., Mueschenborn, S. S., Banting, G., Ratcliff, R., Evans, M. J. \& Colledge, W. H. (1998). Salmonella typhi uses CFTR to enter intestinal epithelial cells. Nature 393, 79-82.

Ravichandran, K. S. (2001). Signaling via Shc family adapter proteins. Oncogene 20, 6322-6330.

Rivera, G. M., Antoku, S., Gelkop, S., Shin, N. Y., Hanks, S. K., Pawson, T. \& Mayer, B. J. (2006). Requirement of Nck adaptors for actin dynamics and cell migration stimulated by platelet-derived growth factor B. Proc Natl Acad Sci U S A 103, 9536-9541.

Rohatgi, R., Nollau, P., Ho, H. Y., Kirschner, M. W. \& Mayer, B. J. (2001). Nck and phosphatidylinositol 4,5-bisphosphate synergistically activate actin polymerization through the N-WASP-Arp2/3 pathway. J Biol Chem 276, 26448-26452.

Schlumberger, M. C. \& Hardt, W. D. (2006). Salmonella type III secretion effectors: pulling the host cell's strings. Curr Opin Microbiol 9, 46-54.

Shi, J. \& Casanova, J. E. (2006). Invasion of host cells by Salmonella typhimurium requires focal adhesion kinase and p130Cas. Mol Biol Cell 17, 4698-4708.

Shi, J., Scita, G. \& Casanova, J. E. (2005). WAVE2 signaling mediates invasion of polarized epithelial cells by Salmonella typhimurium. J Biol Chem 280, 29849-29855.

Soldati, T. \& Schliwa, M. (2006). Powering membrane traffic in endocytosis and recycling. Nat Rev Mol Cell Biol 7, 897-908.

Stanasila, L., Abuin, L., Diviani, D. \& Cotecchia, S. (2006). Ezrin directly interacts with the $\alpha 1 \mathrm{~b}$-adrenergic receptor and plays a role in receptor recycling. J Biol Chem 281, 4354-4363.

Stender, S., Friebel, A., Linder, S., Rohde, M., Mirold, S. \& Hardt, W. D. (2000). Identification of SopE2 from Salmonella typhimurium, a conserved guanine nucleotide exchange factor for Cdc42 of the host cell. Mol Microbiol 36, 1206-1221.

Tanaka, M., Gupta, R. \& Mayer, B. J. (1995). Differential inhibition of signaling pathways by dominant-negative $\mathrm{SH} 2 / \mathrm{SH} 3$ adapter proteins. Mol Cell Biol 15, 6829-6837.

Tomasevic, N., Jia, Z., Russell, A., Fujii, T., Hartman, J. J., Clancy, S., Wang, M., Beraud, C., Wood, K. W. \& Sakowicz, R. (2007). Differential 
regulation of WASP and N-WASP by Cdc42, Rac1, Nck, and PI(4,5)P2. Biochemistry 46, 3494-3502.

Unsworth, K. E., Way, M., McNiven, M., Machesky, L. \& Holden, D. W. (2004). Analysis of the mechanisms of Salmonella-induced actin assembly during invasion of host cells and intracellular replication. Cell Microbiol 6, 1041-1055.

Wray, C. \& Sojka, W. J. (1978). Experimental Salmonella typhimurium infection in calves. Res Vet Sci 25, 139-143.
Yan, Q., Sun, W., Kujala, P., Lotfi, Y., Vida, T. A. \& Bean, A. J. (2005). CART: an Hrs/actinin-4/BERP/myosin V protein complex required for efficient receptor recycling. Mol Biol Cell 16, 2470-2482.

Zhou, D., Chen, L. M., Hernandez, L., Shears, S. B. \& Galan, J. E. (2001). A Salmonella inositol polyphosphatase acts in conjunction with other bacterial effectors to promote host cell actin cytoskeleton rearrangements and bacterial internalization. Mol Microbiol 39, 248-259.

Edited by: P. H. Everest 